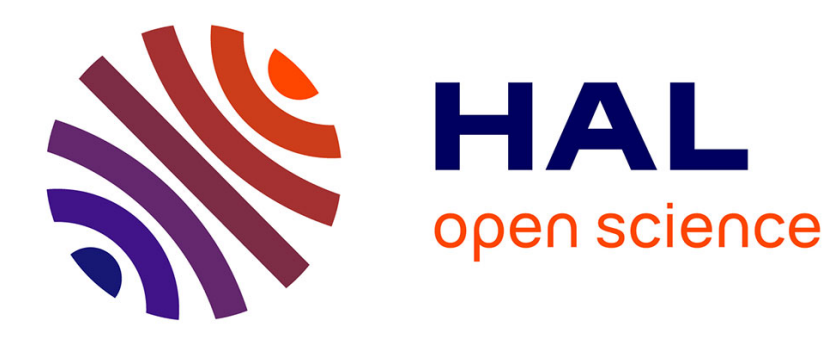

\title{
Positivity constraints on spin observables in hadronic inclusive reactions
}

Claude Bourrely, Jacques Soffer

\section{To cite this version:}

Claude Bourrely, Jacques Soffer. Positivity constraints on spin observables in hadronic inclusive reactions. Physical Review D, 2007, 75, pp.117501. hal-00144864v2

\section{HAL Id: hal-00144864 \\ https://hal.science/hal-00144864v2}

Submitted on 7 May 2007

HAL is a multi-disciplinary open access archive for the deposit and dissemination of scientific research documents, whether they are published or not. The documents may come from teaching and research institutions in France or abroad, or from public or private research centers.
L'archive ouverte pluridisciplinaire HAL, est destinée au dépôt et à la diffusion de documents scientifiques de niveau recherche, publiés ou non, émanant des établissements d'enseignement et de recherche français ou étrangers, des laboratoires publics ou privés. 


\title{
Positivity constraints on spin observables in hadronic inclusive reactions.
}

\author{
Claude Bourrely* \\ Centre de Physique Théorique ${ }^{\dagger}$, \\ CNRS Luminy case 90\%, \\ F-13288 Marseille Cedex 09, France \\ Jacques Soffer $\ddagger$
}

Department of Physics, Temple University, Philadelphia, PA 19122-6082, USA

\begin{abstract}
We study the implications on experimental data and theoretical models of the positivity constraints on spin observables for the reaction $\mathrm{A}(\operatorname{spin} 1 / 2)+\mathrm{B}($ unpolarized $) \rightarrow \mathrm{C}(\operatorname{spin} 1 / 2)$ + anything.
\end{abstract}

PACS numbers: 13.88.+e, 13.85.Ni, 13.75.Ev

The positivity constraints have been widely used in hadron physics to restrict, in a modelindependent way, the domain of allowed values for spin observables $[1,2]$. For a parity conserving inclusive reaction of the type,

$$
\begin{aligned}
& A(\text { spin } 1 / 2)+B(\text { unpolarized }) \\
& \rightarrow C(\text { spin } 1 / 2)+X
\end{aligned}
$$

one can define eight independent observables, which depend on three kinematic variables, $\sqrt{s}$ the center-of-mass energy and $x_{F}, p_{T}$ the longitudinal and transverse momenta of the final particle C. In order to define these observables, we recall the standard notation already used in Ref. [1](Appendix 3), $(A B \mid C X)$, by which the spin directions of $A, B, C$ and $X$ are specified, in one of the three possible directions $L, N, S$. Since only one initial and one final spin are observed, we have in fact $(A 0 \mid C 0)$ and $\mathbf{L}, \mathbf{N}, \mathbf{S}$ are unit vectors, in the center-of-mass system, along the incident momentum, along the normal to the scattering plane which contains $A, B$ and $C$, and along $\mathbf{N} \times \mathbf{L}$, respectively. In addition to the unpolarized cross section $\sigma_{0}=(00 \mid 00)$, there are seven spin dependent observables, two single

\footnotetext{
*Electronic address: Claude.Bourrely@cpt.univ-mrs.fr ${ }^{\dagger}$ Unité Mixte de Recherche 6207 du CNRS et des Universités Aix-Marseille I, Aix-Marseille II et de l'Université du Sud Toulon-Var - Laboratoire affilié à la FRUMAM. ${ }^{\ddagger}$ Electronic address: jacques.soffer@gmail.com
}

transverse spin asymmetries

$$
A_{N}=(N 0 \mid 00) \text { and } P_{C}=(00 \mid N 0),
$$

and five depolarization parameters

$$
\begin{aligned}
& D_{L L}=(L 0 \mid L 0), D_{S S}=(S 0 \mid S 0), \\
& D_{L S}=(L 0 \mid S 0), D_{N N}=(N 0 \mid N 0), \\
& \text { and } D_{S L}=(S 0 \mid L 0) .
\end{aligned}
$$

Several years ago, Doncel and Mèndez [3] have derived very general inequalities constraining these parameters which read

$$
\begin{aligned}
\left(1 \pm D_{N N}\right)^{2} \geq & \left(P_{C} \pm A_{N}\right)^{2}+\left(D_{L L} \pm D_{S S}\right)^{2} \\
& +\left(D_{L S} \mp D_{S L}\right)^{2} .
\end{aligned}
$$

Let us first concentrate on a particular reaction $p^{\uparrow} p \rightarrow \Lambda^{\uparrow} X$, where the incoming proton beam is polarized and the polarization of the outgoing $\Lambda$ is measured. We consider the case where the particle spins are normal to the scattering plane, then the inequalities (4) give

$$
1 \pm D_{N N} \geq\left|P_{\Lambda} \pm A_{N}\right|
$$

these constraints must be satisfied for any kinematic values of the variables $x_{F}, p_{T}, \sqrt{s}$.

These inequalities involve three spin parameters, once we fixe the value of one parameter, the other two are restricted to lie in a certain domain. For instance, Fig. 1 shows that for $D_{N N}=0, P_{\Lambda}$ and $A_{N}$ are correlated within the shaded area of a square with boundaries $(-1,+1)$. 
This domain is more restricted when $D_{N N}=1 / 3$ ( see Fig. 1) and in the limit $D_{N N}=1$, we immediately deduce from Eq. (5) that $P_{\Lambda}=A_{N}$. In the case where $D_{N N}$ is negative, one obtains the same regions, but $P_{\Lambda}$ and $A_{N}$ are interchanged with respect to their axes.

Various applications can be envisaged with the above inequalities, in particular testing, on the one hand, the consistency of experimental data and, on the other hand, the validity of the spin observables predicted by theoretical models.
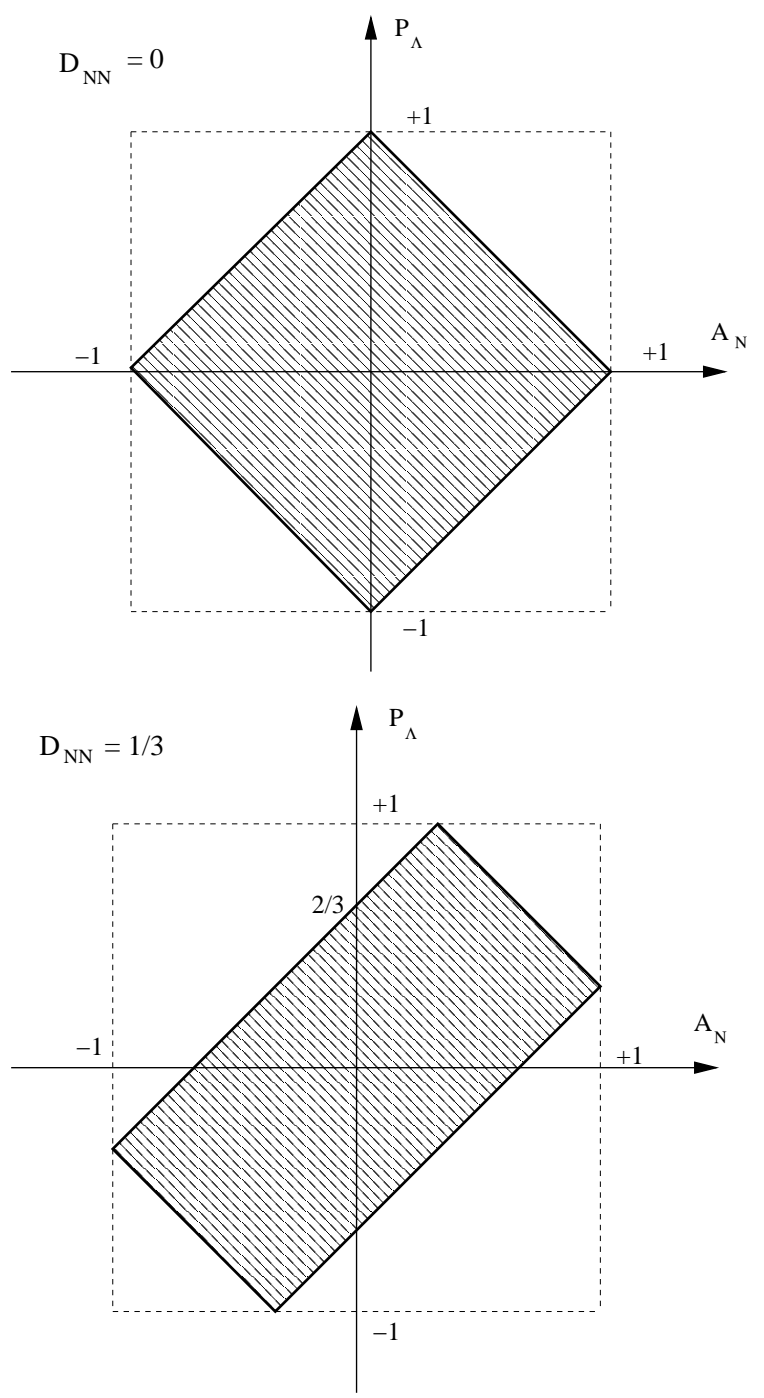

FIG. 1: The polarization $P_{\Lambda}$ versus the analyzing power $A_{N}$ in the cases $D_{N N}=0,1 / 3$.

At FNAL, the experiment E-704 [4] has performed the measurement of $P_{\Lambda}, A_{N}$ and $D_{N N}$ with transversely polarized proton beam at 200 $\mathrm{GeV} / \mathrm{c}$, in the kinematic range $0.2 \leq x_{F} \leq 1.0$ and $0.1 \leq p_{T} \leq 1.5 \mathrm{GeV} / \mathrm{c}$. Their data indicate a negative $A_{N}$ and a value of $D_{N N}$ up to $30 \%$. If we take for instance $p_{T} \sim 1 \mathrm{GeV} / \mathrm{c}, x_{F} \sim 0.8$, then $D_{N N} \sim 30 \%$, for these kinematical values, they have $A_{N} \sim-10 \%$ and $P_{\Lambda} \sim-30 \%$. In this case the inequalities (5) are well satisfied.

The DISTO Collaboration [5] has measured the $A_{N}$ and $D_{N N}$ parameters for three beam momenta $3.67,3.31$ and $2.94 \mathrm{GeV} / \mathrm{c}$ as a function of $x_{F}, p_{T}$, for the exclusive reaction $p^{\uparrow} p \rightarrow p K^{+} \Lambda^{\uparrow}$. In the range $-0.6 \leq x_{F} \leq 0.8$, they find $D_{N N} \approx-50 \%$ and $-15 \% \leq A_{N} \leq 0$, so by using the above inequalities we obtain the constraint $-35 \% \leq P_{\Lambda} \leq 50 \%$. They also have [6] at 3.67 $\mathrm{GeV} / \mathrm{c}$ and $x_{F}=-0.45, D_{N N}=-0.57 \pm 0.07$ and $A_{N}=-0.27 \pm 0.02$, which gives a stronger constraint on $P_{\Lambda}$.

Finally, let us mention the measurement of $D_{N N}$ in the inclusive process $p^{\uparrow} p \rightarrow p^{\uparrow} X$ at $6 \mathrm{GeV} / \mathrm{c}$ incident momentum [7]. For the momentum-transfer $-t=0.20 \mathrm{GeV}^{2}, D_{N N}$ was found rather large and close to +1 , so positivity implies $P_{p} \sim A_{N}$, as indicated above. Unfortunately the data is unavailable, so we were not able to check this expectation.

We see that these constraints are useful to check experimental data, which must lie inside the domain allowed by positivity.

On the theoretical side, different phenomenological models have been proposed to understand the important polarization effects in hyperon production [8]. In Ref. [9], a semiclassical picture is proposed where the direction of the orbital motion of the polarized valence quarks in the initial proton induces the left-right asymmetry for inclusive $\Lambda$. The calculations lead to a reasonable agreement for $P_{\Lambda}, A_{N}$ and $D_{N N}$ in the inclusive $\Lambda$ production, which satisfy the positivity constraints. This model was also applied successfully to the diffractive process $p p \rightarrow \Lambda K^{+} p$ with $D_{N N}=+1$, which implies $P_{\Lambda}=A_{N}$ [10].

When the polarization is not normal to the scattering plane, one has new depolarization parameters. In the case where both $p$ and $\Lambda$ are longitudinally polarized, one can measure $D_{L L}$ and when each particle has a transverse polarization in the scattering plane, one can measure $D_{S S}$. In this case, one get the non-trivial constraint

$$
1 \pm D_{N N} \geq\left|D_{L L} \pm D_{S S}\right| .
$$


Note that the two depolarization parameters in the right hand side of Eq. (6) have not been measured. The BNL-RHIC $p p$ collider is certainly the most appropriate machine for these experiments at high energy, due to the existence of both longitudinally and transversely polarized proton beams. Positivity was used to determine the allowed domains for $D_{L L}[11]$ and $D_{N N}$ [12].
[1] C. Bourrely, E. Leader and J. Soffer, Phys. Rep. 59, 95 (1980).

[2] For a review, see X. Artru, M. Elchikh, J.-M. Richard, J. Soffer and O. Teryaev, Phys. Rep. (in preparation).

[3] M.R. Doncel and A. Méndez, Phys. Lett. B 41, 83 (1972).

[4] Fermilab E-704 Collaboration, A. Bravar et al., Phys. Rev. Lett. 75, 3073 (1995); 78, 4003 (1997). See also the review by L.G. Pondrom, Phys. Rep. 122, 57 (1985).

[5] DISTO Collaboration, R. Bertini, Czechoslovak J. Phys. 51, A77 (2001).
[6] R. Bertini, private communication.

[7] H. Courant et al., Phys. Rev. Lett. 44, 1373 (1980).

[8] J. Félix, Mod. Phys. Lett. A 14, 827 (1999).

[9] C. Boros and Liang Zuo-tang, Phys. Rev. D 53, R2279 (1996) ; Phys. Rev. Lett. 79, 3608 (1997).

[10] C. Boros and Liang Zuo-tang, Phys. Rev. D 61, 117503 (2000).

[11] D. de Florian, M. Stratman and W. Vogelsang, Phys. Rev. Lett. 81, 530 (1998).

[12] D. de Florian, J. Soffer, M. Stratman and W. Vogelsang, Phys. Lett. B 439, 176 (1998). 